\title{
Heart Disease Prediction System Using Supervised Learning Classifier
}

\author{
R. Chitra and Dr.V. Seenivasagam
}

\begin{abstract}
Cardiovascular disease remains the biggest cause of deaths worldwide and the Heart Disease Prediction at the early stage is importance. In this paper Supervised Learning Algorithm is adopted for heart disease prediction at the early stage using the patient's medical record is proposed and the results are compared with the known supervised classifier Support Vector Machine (SVM). The information in the patient record is classified using a Cascaded Neural Network (CNN) classifier. In the classification stage 13 attributes are given as input to the CNN classifier to determine the risk of heart disease. The proposed system will provide an aid for the physicians to diagnosis the disease in a more efficient way. The efficiency of the classifier is tested using the records collected from 270 patients. The results show the CNN classifier can predict the likelihood of patients with heart disease in a more efficient way.
\end{abstract}

Keywords--- Cascaded Neural Network, Heart Disease Prediction, Support Vector Machine, Supervised Learning Algorithm

\section{INTRODUCTION}

$\mathrm{T}$ HE analysis of disease is a vital job in medicine. The health care industry collects huge amount of healthcare data and then they are mined to discover hidden information for effective decision making. Cardiovascular disease is a kind of serious health imperiling and frequent happening disease. Cardiovascular diseases refer to any disease that affects the cardiovascular system. Medical diagnosis is considered a significant task that needs to be carried out precisely and efficiently. The automation of the same would be highly beneficial.

An intelligent heart disease prediction system built with the aid of data mining technique like decision trees, naïve bayed and neural network was proposed by Sellappan Palaniappan et al.[4]. The result illustrated the peculiar strength of each of the methodologies in comprehending the objectives of the specified mining objectives. It facilitated the establishment of vital knowledge eg. patterns connected with heart disease. It subsets wellbeing web-based, user-friendly, scalable, reliable and expandable Markos G. Tsipouras et al[6]

R. Chitra, Associate Professor, Department of Computer Science and Engineering, NI University, Kanyakumari District, India. E-mail: jesi_chit@yahoo.co.in

Dr.V. Seenivasagam, Professor, Department of Computer Science and Engineering, National Engineering College, Kovilpatti, India. E-mail: yespee1094@yahoo.com

DOI: 10.9756/BIJSESC.4336 presented a fuzzy rule based decision support system for the diagnosis of coronary artery disease.

Fuzzy model was used to optimize the parameters. Shanthakumar et al[2] introduced K-means clustering algorithm to extract the data appropriate to heart attack from the warehouse.

In addition the pattern vital to heart attack were selected on basis of the computer significant weight age.

Carlos Ordonez[3] used association rules to improve heart disease prediction. Association rules were plied on a real data set contacting medical records of patient with heart disease and the risk factors were identified. K.Srinivas et al[5] applied data mining techniques to predict heart attack. Using medical profiles such as age, sex, blood pressure and blood sugar it can predict the likelihood of patients getting a heart attack. Based on the calculated significant weight age the frequent pattern having value greater than a predefined threshold were chosen for the valuable prediction of heart attack.

Resul das et [13] al proposed neural network ensembles to predict heart disease. The ensemble based methods creates new models by combining the existing methods to produce the new model for disease prediction. Three independent neural networks models were used to construct the ensemble model. The number of neural networks node in the ensemble model was also increased but no performance improvement was obtained.

Latha Parthiban et al proposed a new approach based on coactive neuro-fuzzy inference system for prediction of heart disease. The proposed CANFIS model combined the neural network adaptive capabilities and the fuzzy logic qualitative approach which is then integrated with genetic algorithm to diagnose the presence of the disease. The performances of the developed model were evaluated in terms of training performances and classification accuracies.

Niti et al. in their work proposed a decision support system for heart disease diagnosis using neural network. They trained their system with 78 patient records and the errors made by humans are avoided in this system[14].

The Support Vector Machine (SVM) is a supervised learning technique introduced first by Vapnik and has been applied in several applications recently, mainly in the area of time series prediction and classification [17]. SVM has yielded excellent generalization performance on a wide range of problems including bioinformatics, text categorization, image detection, micro-array gene expression data analysis, tone recognition, etc. 
The main objective of this research is to develop a Intelligent Heart Disease Prediction System using CNN with back propagation training algorithm using historical heart disease databases to make intelligent clinical decisions which traditional decision support systems cannot. Several computer aided diagnosis methodologies have been proposed in the literature for the diagnosis of heart attacks. An intelligent heart disease prediction system built with the aid of data mining technique like decision trees, naive byes and neural network was proposed by many researchers. The result illustrated the peculiar strength of each of the methodologies in comprehending the objectives of the specified mining objectives. It facilitated the establishment of vital knowledge eg. patterns connected with heart disease.

In this paper, an adaptive intelligent mechanism using CNN, developed by Fahlman\&Lebiere [11] andis adopted to detect the cardiovascular disease at the early stage. CNN has been proved to be a well know decision support system for prediction in nonlinear applications [1]. Artificial Neural Network (ANN) has the capability to learn complex problem in nonlinear environments. Although many ANN techniques are successfully applied to heart attack prediction system, the fixed number of hidden neurons has not been well practice. Hence we adopt a novel methodology to predict the cardiovascular disease by determining the size and topology of the network automatically. A cascade neural network consists of a cascade architecture, in which hidden neurons are added to the network one at a time and do not change after they have been added. The architecture learns very quickly since it requires no back propagation of error signals through the connections of the network. The performance of the developed model is compared with SVM classifier.

In the proposed work the supervised learning method is adopted for this classifier.CNN and SVM classifier are initially trained with known target and then only the actual classification is carried out.

The organization of the paper is as follows. In the second section, the Dataset and the features that are used in this work are described. The architecture of CNN and implementation of CNN classifier for heart disease prediction is presented in the third section. In the fourth section the heart disease prediction system using SVM classifier is explained. The performance of CNN and SVM are analyzed in the fifth section. Concluding remarks and suggestions for possible future work are given in the last section.

\section{MATERIALS AND METHODS}

Many techniques are adapted for predicting cardio vascular diseases. In this proposed work CNN is used to predict the risk of heart attack. The rest of the paper described the dataset and methodology used for heart attack prediction.

In computer-aided heart disease diagnosis methods, where the data is obtained from some other sources and is evaluated by computer based applications. Computers have usually been used to build knowledge based clinical decision support systems which used the knowledge from medical experts, and transferring this knowledge into computer algorithms was done manually. This process is time consuming and really depends on the medical expert's opinion, which may be subjective. To handle this problem, machine learning techniques have been developed to gain knowledge automatically from examples or raw data. Medical diagnosis is an important but complicated task that should be performed accurately and efficiently and its automation would be very useful.

The methodology that is proposed for the Heart Attack Prediction is presented in Fig.1

\section{A. Data Set}

Data used for proposed system is obtained from UCI (University of California, Irvine C.A) Centre for machine learning and intelligent systems. The data have been collected from 270 patients are used for proposed work. This database contains 76 attributes, but experiment refers to use 13 of them. Cleaning and filtering of the data set is done to remove duplicate records, normalize the values, accounting for missing data and removing irrelevant data items.

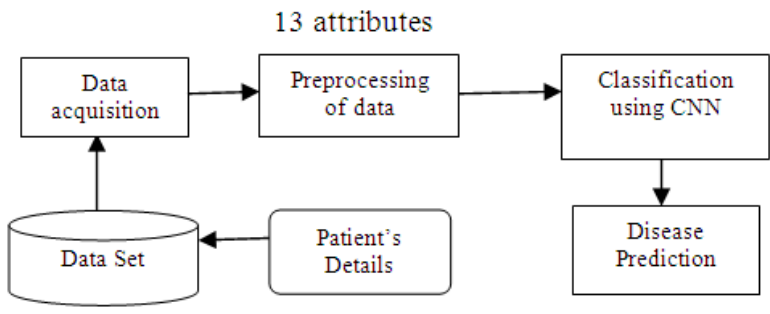

Figure 1: Block Diagram of the Proposed System

Characteristics of the patients like number of times of chest pain and age in years were recorded. In Table 1, there are 13 attributes used in this system, including 8 symbolic and 6 numeric attributes

Some other important parameters are variable parameters and that need to be checked for every 2 hours the latch (maximum heart rate achieved), blood pressure $(\mathrm{mm} \mathrm{Hg})$, serum cholesterol in $(\mathrm{mg} / \mathrm{dl})$, and electrocardiographic result. In real world, data is not always complete and in the case of the medical data, it is always true. To remove the number of inconsistencies which are associated with data we use Data preprocessing. Table 1 describes the input attributes used for prediction. 
Table 1: Attributes of Heart Disease Data Sets

1. Age in year

2. Sex (value 1: Male; value 0 :Female)

3. Chest pain type (value 1:typical type 1 angina; value 2 : typical type angina; value 3: non-angina pain; value 4 : asymptomatic)

4. Resting blood pressure ( $\mathrm{mm} \mathrm{Hg}$ on admission to the hospital)

5. Serum Cholesterol in $\mathrm{mg} / \mathrm{dl}$

6. Fasting blood sugar (value 1:>120 mg/dl; value $0:<$ $120 \mathrm{mg} / \mathrm{dl}$ )

7. Resting Electrocardiographic results (values 0:normal;value1: 1 having ST-T wave abnormality; value 2:showing probable or definite left ventricular hypertrophy)

8. Maximum heart rate achieved

9. Exercise induced angina (value 1:yes; value $0:$ no)

10. Old peak $=\mathrm{ST}$ depression induced by exercise relative to rest

11. The slope of the peak exercise ST segment (value 1: unsloping; value 2 : flat; value 3 :down sloping)

12. Number of major vessels colored by fluoroscopy (value $0-3$ )

13. Thal ( value 3 = normal; value $6=$ fixed defect; value $7=$ reversible defect )

\section{CNN CLASSIFIER}

Artificial Neural Network can be used to tackle the said problem of prediction in medical dataset involving multiple inputs. Artificial neural networks are inspired in the biological neural nets and are used for complex and difficult tasks. ANN are capable of generalization and hence the classification is natural for them. The advantage of ANNs includes automatic learning of dependencies only from measured data without any need to add further information. The main drawback with the ANN structure is the fixed size of the neurons in the hidden layer. Because of this disadvantage, the network is more likely to get trapped in local minima, making it entirely dependent on initial configuration of network structure.

This section describes about the CNN classifier, its training and the role of $\mathrm{CNN}$ classifier for heart disease prediction.

\section{A. Cascaded Neural Network}

A CNN consists of a cascade architecture, in which hidden neurons are added to the network one at a time and do not change after they have been added. It is called a cascade because the output from all neurons already in the network feed into new neurons. As new neurons are added to the hidden layer, the learning algorithm attempts to maximize the magnitude of the correlation between the new neurons output and the residual error of the network which we are trying to minimize.

CNN are "self-organizing" networks. Cascade correlation network training is quite robust, and good results usually can be obtained with little or no adjustment of parameters [1]. The network begins with only input and output neurons. During the training process, neurons are selected from a pool of candidates and added to the hidden layer.

CNN consists of a layer of input units, one or more layers of hidden units, and one output layer of units. The number of input and output units depends upon the application and requires experimentation to determine the best number of hidden units.

A vector of predictor variable values $x_{i}, x_{2}, \ldots \ldots . . . . x_{p}$, ispresented to the input layer. In addition to the predictor variables, there is a constant input of 1.0, called the bias that is fed to each of the hidden and output neurons; the biasis multiplied by a weight and added to the sum going into the neuron.

For regression type of problems there is only a single neuron in the output layer. Each output neuron receives values from all of the input neurons and all of the hidden layer neurons. For classification problems, a sigmoid transfer function is used as the activation function.

The network begins with an input and the output unit and no hidden units. The number of inputs $\left(I_{p}\right)$ and the output $\left(\mathrm{O}_{p}\right)$ is defined by the problem, which is defined as,

$$
\begin{gathered}
I_{p}=\left\{i_{1}, i_{2}, \ldots \ldots, i_{p}\right\} \\
o_{p}=\left\{o_{1}, o_{2}, \ldots ., o_{p}\right\}
\end{gathered}
$$

Every input is connected to the output unit with the weights obtained from the BP algorithm. There is also a bias which is set to +1 . The hidden units are added to the network one by one until the error is minimized or the stopping criterion is reached. Each new hidden unit receives a connection from each of the network's original inputs and also from every preexisting hidden unit. The hidden unit's input weights are frozen at the time the unit is added to the net; only the output connections are trained repeatedly. Each new unit therefore adds a new one-unit "layer" to the network, unless some of its incoming weights happen to be zero. This leads to the creation of very powerful high-order feature detectors. To create a new hidden unit, we begin with a candidate unit that receives trainable input connections from all of the network's external inputs and from all pre-existing hidden units. The goal of this adjustment is to maximize $S$,

$$
S=\sum_{o}\left|\sum_{p}\left(V_{p}-\bar{V}\right)\left(E_{p, o}-\overline{E_{o}}\right)\right|(1)
$$

Where $o$, is the output unit, $p$, number of patterns in the training set, $V_{p}$ is the candidate units value at $p, E_{p, o}$ is the residual error of all the training pattern at the output unit. $\bar{V}$ and $\overline{E_{o}}$ are the values of $V$ and $E_{o}$ averaged over all patterns.

The residual error is calculated using,

$$
E_{p, o}=\left(Y_{p, o}-T_{p, o}\right)(2)
$$

Where $Y_{p, o}$ the actual output at the output unit o is, $T_{p, o}$ is the desired output at the output unit o. 


\section{B. CNN Training}

The training is done using back prop algorithm. Back prop uses a gradient descent method to update the weights. The algorithm used to train the dataset is discussed below,

Step 1: Initialize the input and output units based on the problem defined. The input and the output neurons are fully connected.

Step 2: Train the network with input and output neurons until the residual error $E_{p, o}$ no longer decreases.

Step 3: Select a temporary unit (Candidate unit) connected with the input unit and find the residual error.

Step 4: Train this network unit $S$ (equ. 1) no longer improves.

Step 5: Connect the temporary unit with the output unit and freeze its weights.

Step 6: Train the Input, output and the hidden unit until the residual error is minimized.

Step 7: Repeat the step 2 to step 6 until the net error falls below a given value.

The initial state of the CNN Architecture and the architecture after adding one hidden unit is shown in figure 2.

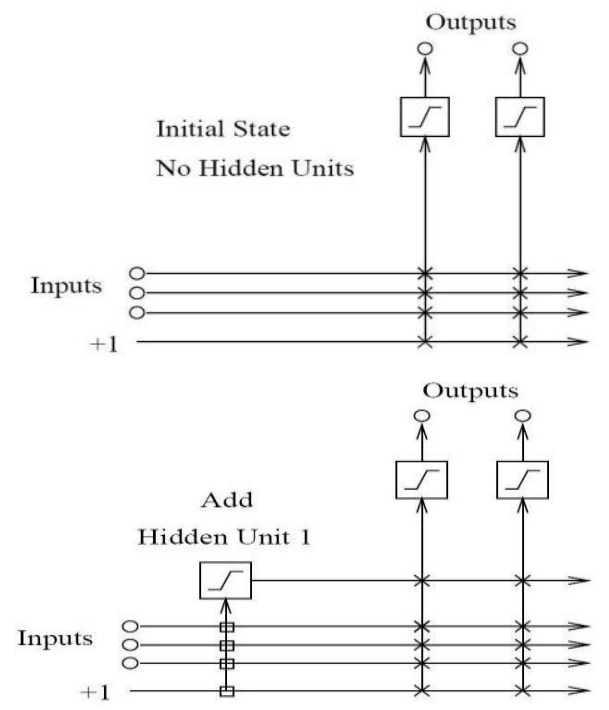

Fig. 2: Cascaded Architecture Structure in Initial State and after adding one Hidden Unit

\section{CNN Based Heart Disease Classification System}

Neural networks can be used for prediction with various levels of success. The advantage of then includes automatic learning of dependencies only from measured data without any need to add further information

The design of the heart disease prediction system with the aid of cascaded neural network is presented in this section. The method primarily based on the information collected from precedent experiences and from current circumstances, which visualizes something as it may occur in future is known as prediction. In this work, a total of 270 data samples are used for simulating the network architecture. 150 samples are used for training the prediction systems and the remaining samples are used for testing the system. The number of input neurons used is $13\left(I_{p}=13\right)$ and the number of output neurons used is 1 $\left(O_{p}=1\right)$.The number of inputs $\left(I_{p}\right)$ and the output $\left(\mathrm{O}_{p}\right)$ is defined as,

$$
\begin{gathered}
I_{p}=\left\{i_{1}, i_{2}, \ldots \ldots, i_{13}\right\} \\
O_{p}=O_{1}
\end{gathered}
$$

The maximum epochs is set to 150 . In case of ANN, training completes at 149 epochs whereas NN takes 117 epochs to reach the desired residual error value.

\section{SVM CLASSIFIER}

\section{A. Support Vector Machine}

SVM is a set of related supervised learning method used in medical diagnosis for classification and regression. SVM simultaneously minimize the empirical classification error and maximize the geometric margin [16]. SVM is called Maximum Margin Classifiers and it can be efficiently perform non-linear classification using kernel trick. An SVM model is a representation of the examples as points in space, mapped so that the examples of the separate categories are divided by a large margin gap that is as wide as possible[17].

Given labeled training data as data points of the form

$$
M=\left\{\left(x_{1}, y_{1}\right),\left(x_{2}, y_{2}\right), \ldots \ldots,\left(x_{n}, y_{n}\right)\right\}
$$

Where $y_{n}=1 /-1$, a constant that denotes the class to which that point $x_{n}$ belongs. $\mathrm{n}=$ number of data sample. Each $x_{n}$ is a p-dimensional real vector. The SVM classifier first maps the input vectors into a decision value, and then performs the classification using an appropriate threshold value. To view the training data, we divide (or separate) the hyperplane, which can be described as:

$$
\text { Mapping: } w^{T} \cdot x+b=0
$$

Where $\mathrm{w}$ is a $\mathrm{p}$-dimensional weight vector and $\mathrm{b}$ is a scalar. The vector $w$ points perpendicular to the separating hyperplane. The offset parameter $b$ allows to increase the margin. In the absence of $b$, the hyperplane is forced to pass through the origin, and restricts the solution.

\section{B. RBF Kernel Function}

The RBF kernel of SVM is used as the Classifier, as RBF kernel function can analyze higher-dimensional data .The output of the kernel is dependent on the Euclidean distance of $x_{j}$ from $x_{i}$. The support vector will be the centre of the RBF and $\gamma$ will determine the area of influence this support vector has over the data space.

In this work RBF kernel function is used because [18] RBF kernel nonlinearly maps samples into a higher dimensional space and also it has less numerical difficulties.

RBF Kernel function can be defined as

$$
\begin{gathered}
k\left(x_{i}, x_{j}\right)=\exp \left(-\gamma|| x_{i}-\left.x_{j}\right|^{2}\right), \\
\gamma>0
\end{gathered}
$$

Where $\gamma$ is a kernel parameter and $x_{i}, x_{j}$ is the training vector. 
A larger value of $\gamma$ will give a smoother decision surface and more regular decision boundary. This is because an RBF with large $\gamma$ will allow a support vector to have a strong influence over a larger area. The best parameter set is applied to the training dataset and classifier is obtained for that. The designed classifier is used to classify the testing dataset to get the generalization accuracy.

\section{Heart Disease Classification using RBF kernel SVM Classifier}

The values fed to the SVM classifier are normalized initially to improve the accuracy. Test data sets were used to assess the performance of the SVM model. Validation using the test data sets avoided potential bias of the performance estimate due to over-fitting of the model to training data sets. The SVM classifier with RBF kernel is used for classification. An automated classifier for the discrimination between the person with heart disease and without heart disease has been developed using supervised learning algorithm named SVM .The heart disease dataset contains 270 data. 100 data are used for training and 170 data are used for testing. The dataset with 13 attributes is fed to the SVM classifier and the output is mapped into two classes normal and abnormal. The choice of best value of parameters for particular kernel is critical for a given amount of data. The classification performance of the heart disease dataset is discussed in the next section.

\section{RESUlt ANALYSIS AND DisCUSSION}

The training and testing accuracy obtained using CNN is $78.55 \%$ and $85 \%$ respectively. The training and testing set accuracy of SVM classifier is $75 \%$ and $82 \%$ respectively. The training and testing set accuracy it is proved that the CNN classifier outperforms the other existing statergies in the literature.Moreover,it is observed that the proposed classifier achieved comparable performance over the testing and training set of all the patient records. The training set accuracy of the CNN classifier is shown in figure 3 .

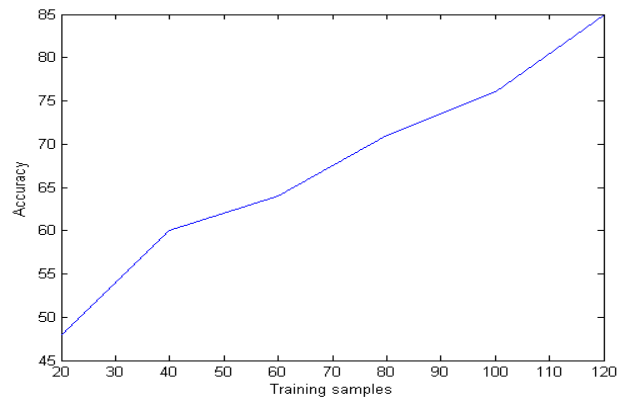

Fig. 3: CNN Training Set Accuracy

In cascaded correlation neural network the epochs got stopped when the desired accuracy got the system will stop immediately. The accuracy of the CNN is $85 \%$ and which is increased almost $3 \%$ compared to SVM. Form figure 3 and 4 it is noticed that the training set accuracy of the CNN classifier is high compared to SVM classifier.

The training set accuracy of proposed SVM classifier is shown in figure 4.

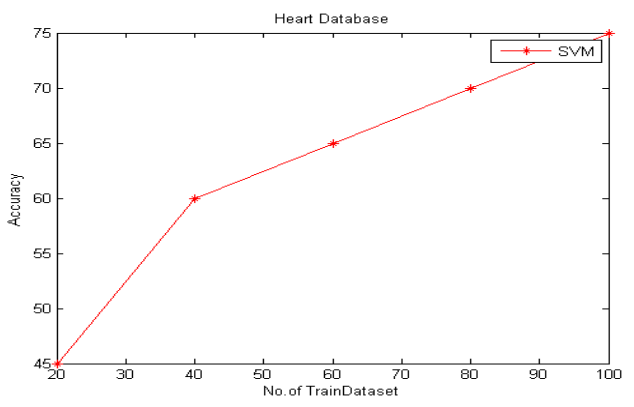

Fig. 4: Training set Accuracy of Heart Disease Dataset using SVM

Sensitivity, specificity and accuracy are the commonly used statistical measures to illustrate the medical diagnostic test and especially, used to enumerate how the test was good and consistent. Sensitivity evaluates the diagnostic test correctly at detecting a positive disease. Specificity measures how the proportion of patients without disease can be correctly ruled out. The performance of the proposed heart attack prediction system is also analyzed by calculating the sensitivity and specificity.

When a single test is performed, the person may in fact have the disease or the person may be disease free. The test result may be positive, indicating the presence of disease, or the test result may be negative, indicating the absence of the disease. The sensitivity of a clinical test refers to the ability of the test to correctly identify those patients with the disease.

A high sensitivity is clearly important where the test is used to identify a serious but treatable disease. The specificity of a clinical test refers to the ability of the test to correctly identify those patients without the disease.

Sensitivity, specificity and accuracy were calculated by the formula given below.

$$
\begin{gathered}
\text { Sensitivity }=\mathrm{TP} /(\mathrm{TP}+\mathrm{FN}) \\
\text { Specificity }=\mathrm{TN} /(\mathrm{TN}+\mathrm{FP}) \\
\text { Accuracy }=(\mathrm{TP}+\mathrm{TN}) /(\mathrm{TP}+\mathrm{FP}+\mathrm{TN}+\mathrm{FN})
\end{gathered}
$$

Table 3 shows the specificity, sensitivity and accuracy of the discussed classifiers. Accuracy measures correctly figured out the diagnostic test by eliminating a given condition.

Table 3: Performance Measures for CNN and SVM

\begin{tabular}{|l|c|c|}
\hline Measures & CNN & SVM \\
\hline Accuracy & 0.85 & 0.82 \\
\hline Sensitivity & 0.83 & 0.855 \\
\hline Specificity & 0.87 & 0.775 \\
\hline
\end{tabular}

The specificity of the CNN classifier is comparatively high compared to SVM and hence the CNN classifier can predict the patient without disease. But the sensitivity of the SVM is little bit high compared to CNN and indicated the ability of disease prediction is high for SVM. But the overall accuracy is high for CNN .Hence the CNN classifier is suggested for heart disease prediction with higher accuracy. 
The performance measures such as Accuracy, specificity and sensitivity in percentage of the CNN classifier is compared with the SVM classifier. The comparative performance measure is shown in fig 5.Itisclearly proved that the proposed system with $\mathrm{CNN}$ gives higher accuracy, specificity and sensitivity.

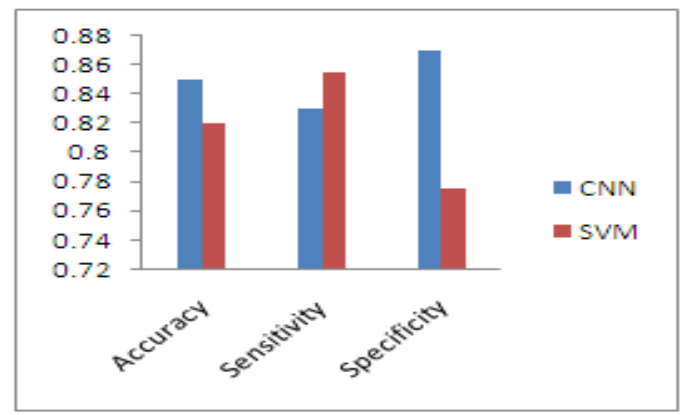

Fig. 5: Performance Measure of SVM and CNN

False Positive Rate and True Positive Rate gives relative trade-off between true positive and false positive.

True Positive Rate $=\mathrm{TP} /(\mathrm{TP}+\mathrm{FN})$

False Positive Rate $=$ FP $/(\mathrm{FP}+\mathrm{TN})$

Table 4 shows True Positive Rate and False Positive Rate for SVM and CNN

Table 4: True Positive Rate and False Positive Rate

\begin{tabular}{|l|l|l|}
\hline & True Positive rate & $\begin{array}{l}\text { False Positive } \\
\text { Rate }\end{array}$ \\
\hline CNN & 0.85 & 0.164 \\
\hline SVM & 0.83 & 0.224 \\
\hline
\end{tabular}

The true positive rate there is a small difference between two classifiers but the false positive rate of $\mathrm{CNN}$ is significantly less compared to SVM classifier. Hence CNN can be suggested for disease prediction in a accurate manner compared with other classifier.

A ROC space is defined by FPR and TPR as $x$ and $y$ axes respectively, which depicts relative trade-offs between true positive and false positive. The best possible prediction method would yield a point in the upper left corner or coordinate $(0,1)$ of the ROC space The ROC space for both classifiers is shown in figure 6.The plot for CNN classifier is shown in filled circle mark.

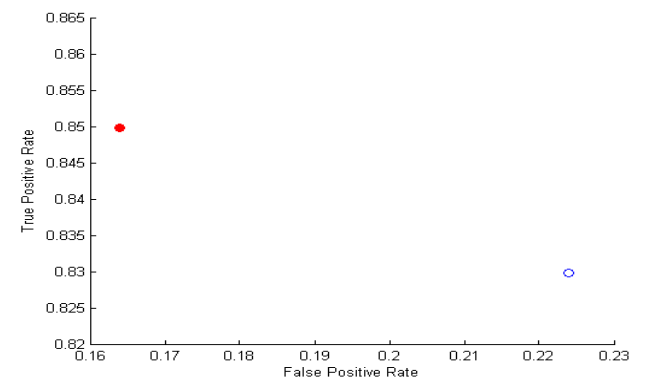

Fig. 6: Plot of ROC Space for SVM and CNN
From the plot of the ROC space shows that for the CNN classifier it lies in the upper left corner and thus the predictive power is high compared to SVM classifier. Hence the CNN is suggested for high power predictive tool for heart disease prediction.

In clinical research a common classification type is a binary classification, which predicts whether a disease is present or not. The performance of the classifiers SVM and CNN is compared based on the accuracy, sensitivity and specificity of the results obtained. For prediction of the disease and for the purpose of comparative analysis the standard heart disease data set taken from California University is used. At first, the attributes are collected from the dataset and then classifiers are built based on the input attributes. The True positive Rate and False Positive Rate is computed for both classifier and the obtained results are shown in Table 4.

When analyzing these graphs in Figure 5 the $\mathrm{CNN}$ classifier shows better performance in terms of accuracy, sensitivity and specificity.CNN has an improved performance than the SVM, this is due to the fact that SVM does not depends on the generalization error.

In summary, it's found that the proposed CNN based prediction system offers substantial improvement in prediction of heart disease. The results of the experiments show that an improvement of $3 \%$ accuracy has been achieved CNN classifier. This implies that the Cascaded Neural Network is one of the desirable classifier, which can be used as an aid for the physicians to predict the heart diseases in a more efficient way.

\section{CONCLUSION}

In this paper, we have proposed Supervised Learning Algorithm for finding the risk of heart disease of a patient using the profiles collected from the patients. The Heart Disease Prediction system using CNN and SVM with Radial basis function kernel is analysed.CNN has a distinct feature that it does not use a predefined set of hidden units, instead the hidden units gets added up one by one until the error is minimized. By exploiting this distinct feature of the CNN, a computerized prediction algorithm is developed that are not only accurate but also computationally efficient for heart attack prediction with the proper adaptation of CNN classifies, the method can thus evolve an optimum number of hidden units within an architecture space. The results of classification experiment, preformed over data sets obtain from 270patients; shows that CNN classifier has achieved better accuracy than SVM classifier.

There are many interesting aspects for future work. In future the performance can be improved by subset selection process Genetic Algorithms can be used to select the input features. Further this system can be enhanced using Swarm intelligence techniques to decide the more weight age input parameters.

\section{REFERENCES}

[1] J. Dheeba and A. Padma, "Intelligent Adaptive Noise Cancellation using Cascaded Correlation Neural Networks "In: IEEE-ICSCN, Pp 178-182, Feb, 2007. 
[2] Shanthakumar B. Patil and Y.S. Kumaraswamy, "Intelligent and Effective Heart Attack Prediction System Using Data Mining and Artificial Neural Network". European Journal of Scientific Research 642-656, Vol. 31, No. 04, 2009.

[3] Carolas Ordonez, "Association Rule Discovery with the Train and Test Approach for Heart Disease Prediction" IEEE Transactions on Information Technology in Biomedicine, Vol. 10, No. 2, April 2006.

[4] Sellappan Palaniappan and Rafiah Awang, "Intelligent Heart Disease Prediction System Using Data Mining Techniques" IEEE, Pp. 108-115; 2008

[5] K. Srinivas, B. Kavitha Rani and Dr.A. Govrdhan "Applications of Data Mining Techniques in Healthcare and Prediction of Heart Attacks" K. Srinivas et al. / (IJCSE) International Journal on Computer Science and Engineering Vol. 02, No. 02, 250-255, 2010

[6] Markos G. Tsipouras, Themis P. Exarchos, Dimitrios I. Fotiadis, Anna P. Kotsia, Konstantinos V. Vakalis, Katerina K. Naka, and Lampros K. Michalis" Automated Diagnosis of Coronary Artery Disease Based on Data Mining and Fuzzy Modeling" IEEE Transactions on Information Technology in Biomedicine, Vol. 12, No. 4, July 2008.

[7] Minas A. Karaolis, Joseph A. Moutiris, DemetraHadjipanayi, and Constantinos S. Pattichis "Assessment of the Risk Factors of Coronary Heart Events Based on Data Mining With Decision Trees IEEE Transactions on Information Technology in Biomedicine, Vol. 14, No. 3, May 2010.

[8] RanjanaRaut and S.V. Dudul "Intelligent Diagnosis of Heart Disease Using Neural Network Approach" International Journal on Computer Applications Engineering Vol. 01, No. 02117-123, 2010.

[9] D. Shanthi, G. Sahoo and N. Saravanan, "Designing an Artificial Neural Network Model for the Prediction of Thrombo-embolic Stroke. International Journal of Biometric and Bioinformatics (IJBB), Vol. 03, No. 01, Pp.10-18, 2009.

[10] Y.S. Kumaraswamy, Shanthakumar B. Pillai" Extraction of Significant Patterns from Heart Disease Warehouses for Heart Attack Prediction" (IJCSNS) International Journal on Computer Science and Network Security, Vol. 09, No. 02, Pp.228-235, February 2009.

[11] S.E. Fahlman and C. Lebiere, "The Cascaded correlation Learning Architecture in Neural Information Processing Systems", Editors D. Touretzky, Morgan Kaufmann Publishers, Inc., Denver, Colorando, Pp. 524-532,1990.

[12] Scott E. Fahlmanand Christian Lebiere, "The cascaded correlation Learning Architecture", Pp. 1-11, August 29, 1991.

[13] Resul Das, Ibrahim Turkoglu, Abdulkadir Sengur, "Effective diagnosis of heart disease through neural networks ensembles "Internationaljournal of expert systems with applications, Vol. 36, Pp. 7675-7680, 2009.

[14] LathaParthiban and R. Subramanian," Intelligent Heart Disease Prediction System using CANFIS and Genetic Algorithm" International Journal of Biological and Life Sciences Vol. 3, No. 3, Pp. 156-160, 2007.

[15] Niti Guru, Anil Dahiya and NavinRajpal, " Decision Support System for Heart Disease Diagnosis using Neural Network", Delhi Business Review,Vol.8, No.1, Pp.99-101, 2007.

[16] Boser, B. E., I. Guyon, and V. Vapnik (1992). A training algorithm for optimal margin Classifiers. In Proceedings of the Fifth Annual Workshop on Computational Learning Theory, Pp. 144 -152. ACM Press. 1992.

[17] Christopher J.C. Burges. A Tutorial on Support Vector Machines for Pattern Recognition. Data Mining and Knowledge Discovery, Springer, 2(2), Pp.121-167, 1998.

[18] V. Vapnik. The Nature of Statistical Learning Theory. NY: Springer Verlag. 1995.

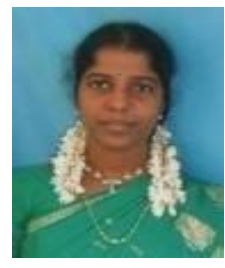

R. Chitra, received the BE degree in Electrical and Electronics Engineering and ME degree in Computer Science and Engineering from Manonmaniam Sundaranar University, India. She is currently working towards the Ph.D degree at the Department of Computer Science and Engineering, Manonmaniam Sundaranar University, India. Her research interests include datamining, intelligent techniques and Bioinformatics. E-mail: jesi_chit@yahoo.co.in

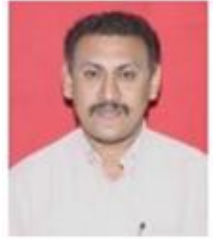

Dr.V. Seenivasagam is presently working as Professor of Computer Science and Engineering at National Engineering College (Autonomous), Kovilpatti and has 23 Years of experience in the field of Computer Science and Engineering. He has published 25 Papers in the fields of image processing and soft computing. He is the member of Board of Studies in the faculty of Computer Science and Engineering at Anna University of Technology, Tirunelveli. His areas of specializations are Image Processing, Compiler Design and Soft Computing. E-mail: yespee1094@yahoo.co.in 